\title{
Heat transfer on rotating second grade fluid through an accelerated plate
}

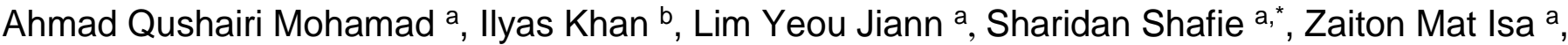 \\ Zulkhibri Ismail c
}

a Department of Mathematical Sciences, Faculty of Science, Universiti Teknologi Malaysia, 81310 UTM Johor Bahru, Johor, Malaysia

${ }^{b}$ College of Engineering Majmaah University, P.O. Box 66, Majmaah 11952, Saudi Arabia

c Faculty of Industrial Science \& Technology, Universiti Malaysia Pahang, 26300, Kuantan, Pahang, Malaysia

*Corresponding author: sharidan@utm.my

\section{Article history}

Received 3 May 2017

Accepted 4 July 2017

\begin{abstract}
Heat transfer on an unsteady free convection rotating second grade fluid flow, which is flowing through an accelerated plate, is analyzed. The physical problem investigated is described by a coupled, linear system of partial differential equations, with appropriate boundary conditions. Laplace transform technique is applied to determine the analytical solutions of the dimensionless governing equations. The effects of various embedded parameters to the velocity and temperature distribution in the fluid are graphically illustrated and analyzed. The obtained analytical results constitute a good verification to verify a more advance situation of the physical problem, which is described by a non-linear system and only can be solved by using approximation method.
\end{abstract}

Keywords: Free convection, second grade fluid, rotating, accelerated, laplace transform

\section{INTRODUCTION}

The investigation of non-Newtonian fluids have received considerable attention due to the wide application in industry, geophysics and engineering. Unlike the Newtonian fluids, numerous models have been developed for these fluids because there is no single model which completely explains all the properties of non-Newtonian fluids (Hayat 2004, 2008, Khan 2010,). Thus, the fluids have been mainly classified under the differential, rate and integral types (Faisal 2013). Due to the non-linear dependence, the analysis of the behaviour of fluid motion of non-Newtonian fluids offer mathematicians, engineers, and numerical specialists varied challenges, and they are developing appropriate analytical and numerical solutions. One of the most popular models for non-Newtonian fluids is second grade fluid. Second grade fluid is the non-Newtonian viscoelastic fluid and commonly used in food mixing and chime movement in the intestine, polymer solutions, paint, flow of plasma, flow of blood, flow of nuclear fuel slurries, the flow of liquid metals and alloys, flow of mercury amalgams, and lubrications with heavy oils and greases (Imran 2014, Azhar 2015). The second grade model is chosen in present study because it can reasonably hope to obtain exact solutions of the model. It is essentials as providing a standard for checking the accuracies of many approximate solutions which obtained numerically or empirically. Moreover, they can also be applied to verify numerical schemes that are developed for studying more complicate flow problems.

Furthermore, the free convection flow in a rotating medium is also important due to its application in many areas of geophysics, astrophysics and fluid engineering Ismail (2015). Studies show that the flow in the earth's liquid core is significantly affected by the Coriolis force due to earth's rotation. Ismail (2015) studied the unsteady MHD free convection flow of a second grade fluid in an infinite inclined plate with a rotating effects. The authors determined an exact solution for the problem and found that the thickness of the boundary layer decreases if the second grade parameter increases. Recently, Mohamad (2016) investigated the unsteady free convection flow of rotating second grade fluids over an oscillating plate. To the best of our knowledge, no literature exists in which accelerated free convection flows of nonNewtonian fluids in rotating frame have been studied.

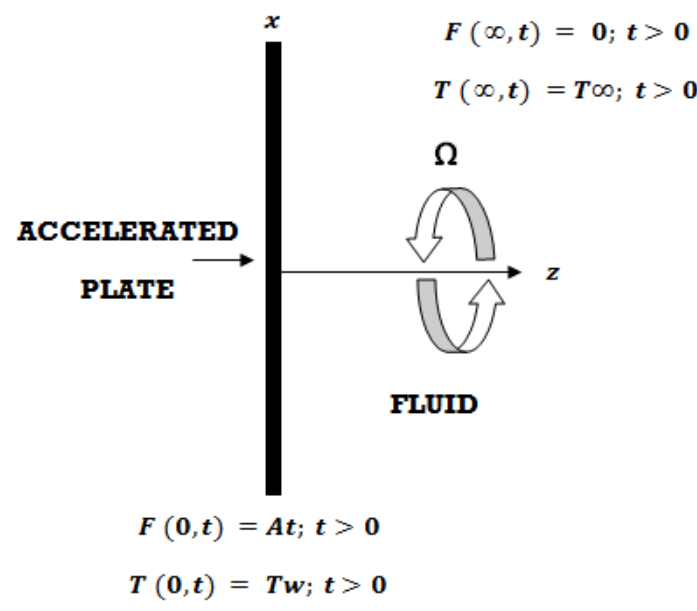

Fig. 1 Physcial diagram and coordinate system. 
Therefore the aim of the present work is to investigate the rotating and accelerated free convection flows in a second grade fluid. All the governing partial differential equations have been solved using Laplace transform. The influence of second grade parameters and rotation effects on the accelerated convection flow are graphically presented and analysed.

\section{MATHEMATICAL FORMULATION AND SOLUTION}

\section{Formulation}

Consider the unsteady incompressible second grade fluid bounded by a rigid moved plate $z=0$. The $z$-axis is taken normal to the plate. Initially, both the plate and fluid are at rest with constant temperature $T_{\infty}$. At time $t=0^{+}$, the plate starts to move with a constant acceleration $A$. After that, the fluid starts solid body rotation with constant angular velocity $\Omega$ parallel to $z$-axis. The dimensional governing momentum and energy equations are

$$
\begin{aligned}
\frac{\partial F}{\partial t}+2 i \Omega F & =v \frac{\partial^{2} F}{\partial z^{2}}+\frac{\alpha_{1}}{\rho} \frac{\partial^{3} F}{\partial z^{2} \partial t}+g \beta\left(T-T_{\infty}\right), \\
\frac{\partial T}{\partial t} & =\frac{k}{\rho c_{p}} \frac{\partial^{2} T}{\partial z^{2}}
\end{aligned}
$$

in which $F=u+\mathrm{i} v$ is the complex veloity where $u$ and $v$ are primary and secondary velocities respectively, $\rho$ is the fluid desity, $v$ is the kinematic viscosity, $\alpha_{l}$ is the second grade parameter, $g$ is the acceleration due to gravity, $\beta$ is the volumetric coefficient of thermal expansion, $T$ is the temperature of the fluid, $k$ is the thermal conductivity and $c_{p}$ is the specific heat capacity of the fluid at constant pressure. The dimensional initial and boundary conditions are

$$
\begin{gathered}
F(0, t)=A t, F(z, t) \rightarrow 0 \text { as } z \rightarrow \infty ; t>0, \\
F(z, 0)=0 ; z>0,
\end{gathered}
$$

and

$$
\begin{gathered}
T(0, t)=T_{w}, T(z, t)=T_{\infty} \text { as } z \rightarrow \infty ; t>0, \\
T(z, 0)=T_{\infty} ; z>0 .
\end{gathered}
$$

The non-dimensionl variables are defined as

$$
F^{*}=\frac{F}{(v A)^{\frac{1}{3}}}, \quad z^{*}=\frac{z A^{\frac{1}{3}}}{v^{\frac{2}{3}}}, \quad t^{*}=\frac{t A^{\frac{2}{3}}}{v^{\frac{1}{3}}}, \quad T^{*}=\frac{T-T_{\infty}}{T_{w}-T_{\infty}} .
$$

\section{Solution}

Substituting Eq. (5) into Eq. (1-4), then obtained non-dimensional equations as follow (dropping out the * notation)

$$
\begin{gathered}
\frac{\partial F}{\partial t}+2 i \omega F=\frac{\partial^{2} F}{\partial z^{2}}+\alpha \frac{\partial^{3} F}{\partial z^{2} \partial t}+G r T, \\
\frac{\partial T}{\partial t}=\frac{1}{\operatorname{Pr}} \frac{\partial^{2} T}{\partial z^{2}}, \\
F(0, t)=t, F(z, t) \rightarrow 0 \text { as } z \rightarrow \infty ; t>0, \\
F(z, 0)=0 ; z>0, \\
T(0, t)=1, T(z, t)=0 \text { as } z \rightarrow \infty ; t>0, \\
T(z, 0)=0 ; z>0,
\end{gathered}
$$

where the parameters involved in this study are

$$
\alpha=\left(\frac{\alpha_{1} A^{\frac{2}{3}}}{\rho v^{\frac{4}{3}}}\right) ; \quad \omega=\frac{\Omega v^{\frac{1}{3}}}{A^{\frac{2}{3}}} ; \quad G r=\frac{g \beta\left(T_{w}-T_{\infty}\right)}{A}, \quad \operatorname{Pr}=\frac{\mu c_{p}}{k},
$$

$\alpha$ is the second grade parameter, $\omega$ is the rotation parameter, $G r$ is the Grashof number and Pr is the Prandtl number. To solve Eq. (6) and Eq. (7) along with initial and boundary conditions (8) and (9), Laplace transform tehcnique has been used. The transformed Eqs. (6) and (7) with conditions (8) and (9) in $q$-domain are

$$
\begin{gathered}
\frac{d^{2} \bar{F}}{d z^{2}}-\left(\frac{q+2 i \omega}{\alpha q+1}\right) \bar{F}=-\frac{G r}{(\alpha q+1)} \bar{T}, \\
\bar{F}(0, q)=\frac{1}{q^{2}}, \quad \bar{F}(z, q) \rightarrow 0 ; \quad \text { as } z \rightarrow \infty,
\end{gathered}
$$

and

$$
\frac{d^{2} \bar{T}}{d z^{2}}-\operatorname{Pr} q \bar{T}=0
$$

$$
\bar{T}(0, q)=\frac{1}{q}, \quad \bar{T}(z, q) \rightarrow 0 ; \quad \text { as } z \rightarrow \infty
$$

Characteristics equation for non-homogenous and homogenous differential equations will be used to solve Eqs. (11) and (13). Solution for Eq. (13) is

$$
\bar{T}(z, q)=c_{1} \exp (-z \sqrt{\operatorname{Pr} q})+c_{2} \exp (z \sqrt{\operatorname{Pr} q})
$$

and using boundary conditions (14), the result is

$$
\bar{T}(z, q)=\frac{1}{q} \exp (-z \sqrt{\operatorname{Pr} q}) .
$$

Substitute Eq. (16) into Eq. (11), given the solution as

$$
\bar{F}(z, q)=\bar{F}_{h}(z, q)+\bar{F}_{p}(z, q),
$$

where

$$
\bar{F}_{h}(z, q)=c_{3} \exp \left(-z \sqrt{a_{1}} \sqrt{\frac{q+2 i \omega}{q+a_{1}}}\right)+c_{4} \exp \left(z \sqrt{a_{1}} \sqrt{\frac{q+2 i \omega}{q+a_{1}}}\right)
$$

and

$$
\bar{F}_{p}(z, q)=-\frac{a_{5}}{q\left[q^{2}+a_{6} q-a_{7}\right]} \exp (-z \sqrt{\operatorname{Pr} q}) .
$$

In order to solve the function (17) subjected to functions (18) and (19), applied the boundary condition into these functions and written as

$$
\begin{aligned}
\bar{F}(z, q)= & \frac{1}{q^{2}} \exp \left(-\sqrt{a_{1}} \sqrt{\frac{q+2 i \omega}{q+a_{1}}}\right)+\frac{a_{5}}{\left[q^{2}+a_{6} q-a_{7}\right]} \times \\
& {\left[\frac{1}{q} \exp \left(-\sqrt{a_{1}} \sqrt{\frac{q+2 i \omega}{q+a_{1}}}\right)-\frac{1}{q} \exp (-z \sqrt{\operatorname{Pr} q})\right] }
\end{aligned}
$$


Here, separated function (20) into two parts of function, which are

$$
\bar{F}(z, q)=\bar{F}_{1}(z, q)+\bar{F}_{2}(z, q)
$$

where

$$
\bar{F}_{1}(z, q)=\frac{1}{q^{2}} \exp \left(-\sqrt{a_{1}} \sqrt{\frac{q+2 i \omega}{q+a_{1}}}\right)
$$

and

$$
\begin{aligned}
\bar{F}_{2}(z, q) & =\frac{a_{5}}{\left[q^{2}+a_{6} q-a_{7}\right]} \times \\
& {\left[\frac{1}{q} \exp \left(-\sqrt{a_{1}} \sqrt{\frac{q+2 i \omega}{q+a_{1}}}\right)-\frac{1}{q} \exp (-z \sqrt{\operatorname{Pr} q})\right] . }
\end{aligned}
$$

Then, the inverse Laplace tranform of Eq. (16) and Eqs. $(21-23)$ are

$$
\begin{gathered}
T(z, t)=\operatorname{erfc}\left(\frac{z \sqrt{\mathrm{Pr}}}{2 \sqrt{t}}\right), \\
F(z, t)=F_{1}(z, t)+F_{2}(z, t), \\
F_{1}(z, t)=t \exp \left(-z \sqrt{a_{1}}\right)+ \\
\frac{z a_{9}}{2 \sqrt{\pi}} \int_{0}^{t} \int_{0}^{\infty} \frac{1}{u \sqrt{s}}(t-s) \times \\
\exp \left(-\frac{a_{1} z^{2}}{4 u}-u-a_{1} s\right) I_{1}\left(2 \sqrt{a_{8} u s}\right) d u d s, \\
F_{2}(z, t)=\int_{0}^{t} F_{21}(t-p) F_{22}(z, p) d p,
\end{gathered}
$$

where

$$
F_{21}(t-p)=b_{3} \sinh \left(b_{2}(t-p)\right) \exp \left(-b_{1}(t-p)\right)
$$

and

$$
\begin{aligned}
F_{22}(z, t)= & \exp \left(-z \sqrt{a_{1}}\right)+ \\
& \frac{z a_{9}}{2 \sqrt{\pi}} \int_{0}^{p} \int_{0}^{\infty} \frac{1}{u \sqrt{s}} \exp \left(-\frac{a_{1} z^{2}}{4 u}-u-a_{1} s\right) \times \\
& I_{1}\left(2 \sqrt{a_{8} u s}\right) d u d s- \\
& \operatorname{erfc}\left(\frac{z \sqrt{\operatorname{Pr}}}{2 \sqrt{p}}\right) .
\end{aligned}
$$

Here, the involved constant parameters are

$$
\begin{aligned}
& a_{1}=\frac{1}{\alpha}, a_{2}=\frac{G r}{\alpha}, a_{3}=a_{1} \operatorname{Pr}-a_{1}, a_{4}=2 i \omega a_{1}, \\
& a_{5}=\frac{a_{2}}{\operatorname{Pr}}, a_{6}=\frac{a_{3}}{\operatorname{Pr}}, a_{7}=\frac{a_{4}}{\operatorname{Pr}}, a_{8}=a_{1}-2 i \omega, \\
& a_{9}=\sqrt{a_{1} a_{8}}, \quad b_{1}=\frac{a_{6}}{2}, b_{2}=\frac{\sqrt{a_{6}^{2}+4 a_{7}}}{2}, b_{3}=\frac{a_{5}}{b_{2}} .
\end{aligned}
$$

\section{RESULTS AND DISCUSSION}

In this study, the analytical solutions of velocity and temperature for the rotating second grade fluid past an accelerated plate are obtained using Laplace transform technique as shown in Eqs. (24 - 29). The results for velocity and temperature profiles are presented graphically to support the present analytical solutions where the effects of the second grade fluid parameter $\alpha$, Grashof number $G r$, rotation parameter $\omega$, time parameter $t$ and Prandtl number Pr on temperature $T$ profile are observed. For this purpose, Figs. (3-7) have been plotted for the case of constant acceleration of the plate. In order to check the accuracy of the present solution, the validation result has been plotted and discussed in Fig. 2. It is found that, the present solution (25) when $G r=0$ and $\operatorname{Pr} \rightarrow$ 0 was identical with Khan (2010) when magnetic parameter $M=0$ and posority effect $K \rightarrow 0$. Hence, the accuracy for validation of the present solution is confirmed.

Fig. 3 shows the effect of $\alpha$ on the primary and secondary velocities. It is found that, the primary velocity increases while the secondary velocity first increases and the decreases when $\alpha$ is increased. In the Fig. 4, it is noted that an increase in $G r$ increasess the velocity profile monotonically for both primary and secondary velocities. This is in accordance with the fact that Grashof number will enhance the bouyance force of the flow and causes the velocity to move faster. It is obvious to see that for larger values of $\omega$, the primary velocity is decreasing but quite opposite behavior was observed for the secondary velocity like in Fig. 5. The effect of parameter $t$ on the flow is also discussed in this problem in Fig. 6. As anticipated, an increase $t$ on velocity profiles will causes them to increase due to increase in energy transfered. Lastly, the behavior of Prandtl number on temperature profiles has been plotted in Fig. 7 where the velocity decreases when the values of Pr increases. This is because, the larger values of Pr, the larger viscous force in fluid flow. Therefore, it will reduce the velocity of the fluid.
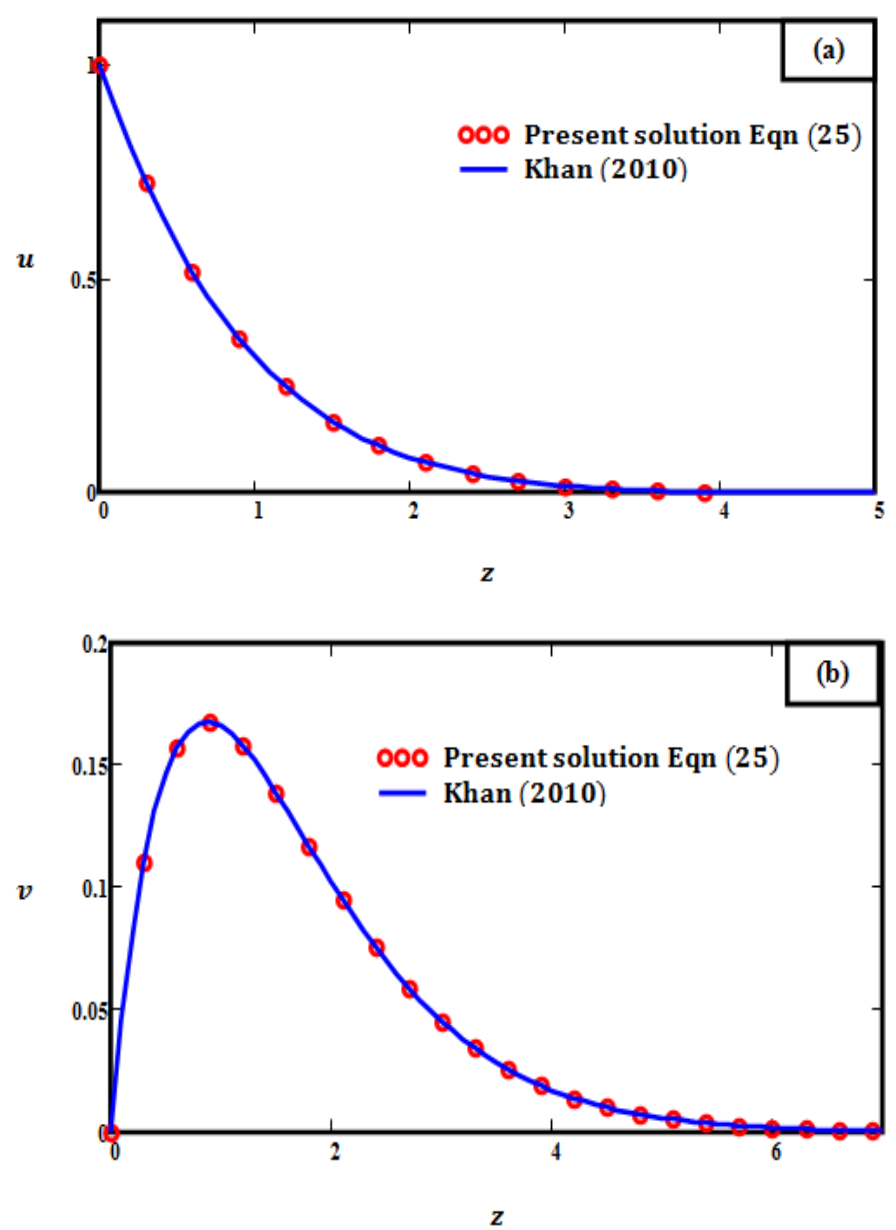

Fig. 2 Validation profiles for present solution (25) and Khan (2010) where (a) primary and (b) secondary velocities. 

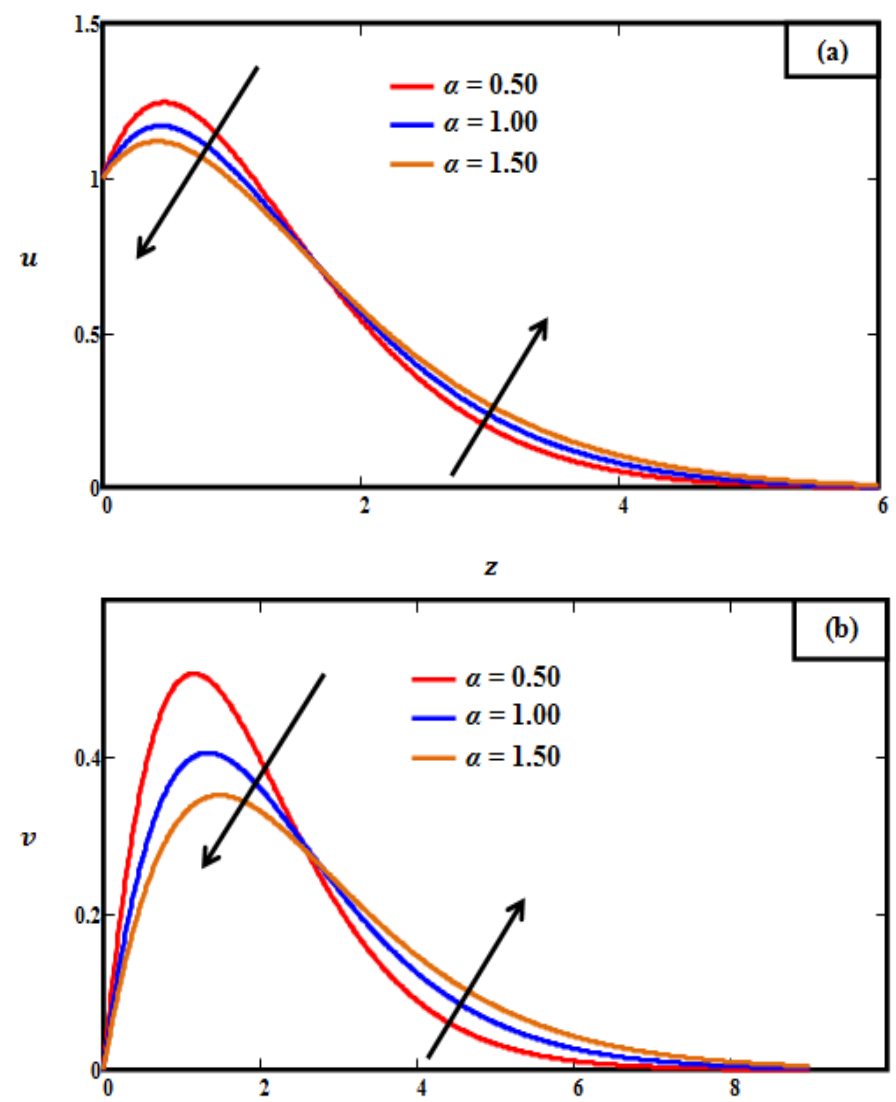

Fig. 3 Velocity profiles for different values of $\alpha$ with $\omega=1.0, t=1.0, G r=5.0$, $\mathrm{Pr}=0.71$ where (a) primary and (b) secondary velocities.
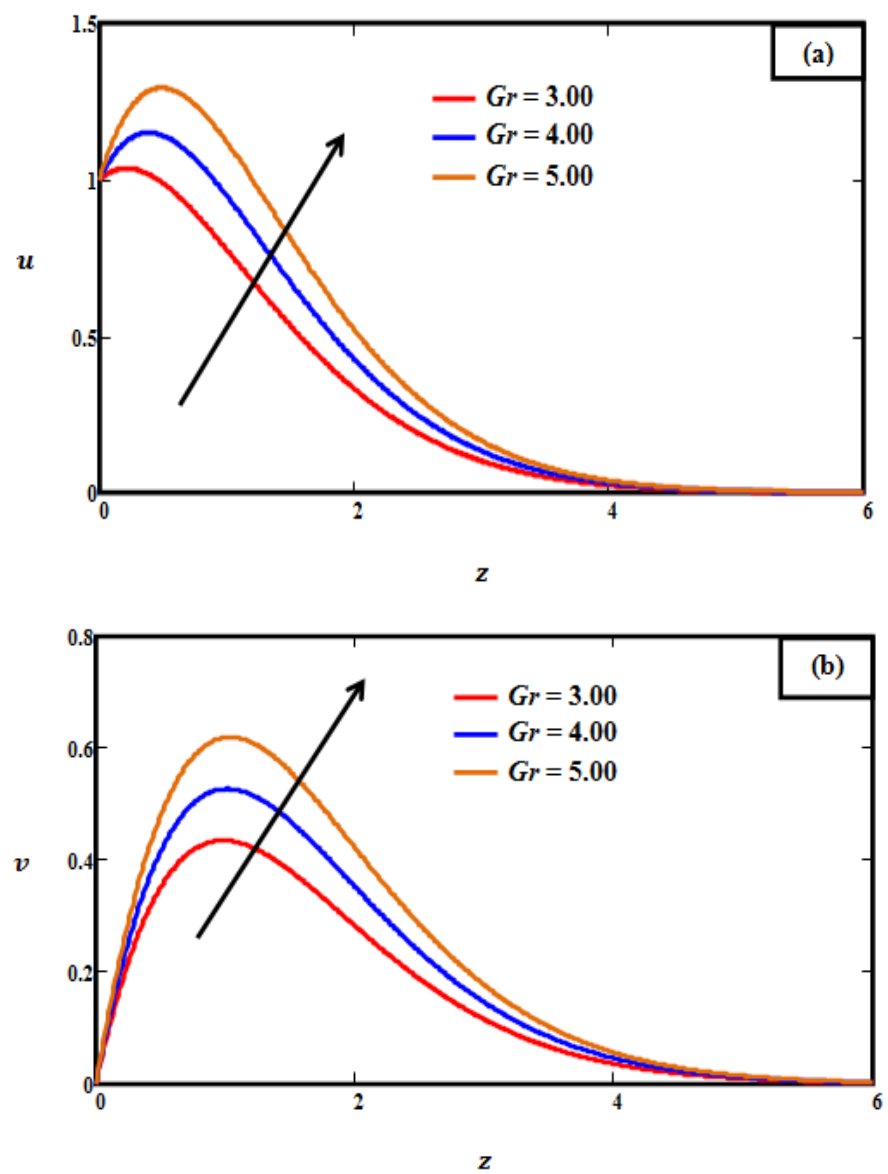

Fig. 4 Velocity profiles for different values of Gr with $\omega=1.0, t=1.0, \alpha=0.2$, $\mathrm{Pr}=0.71$ where (a) primary and (b) secondary velocities.

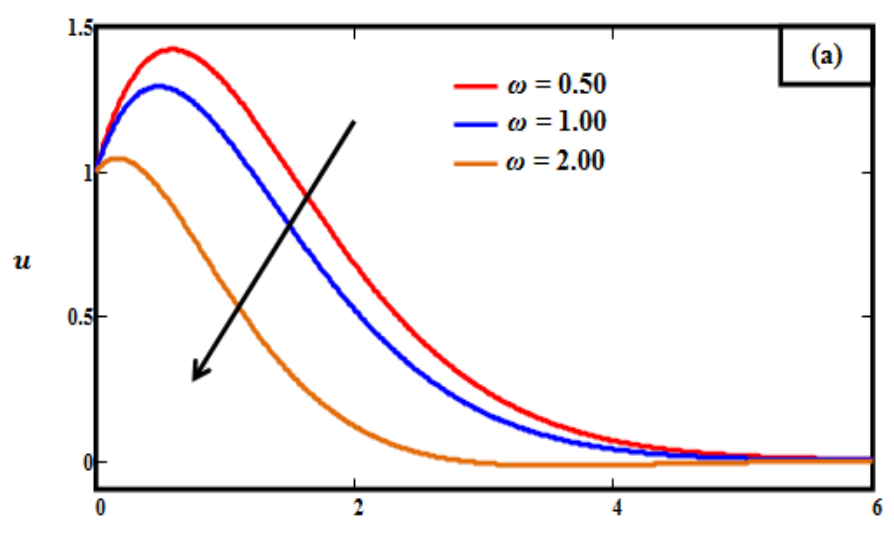

$z$

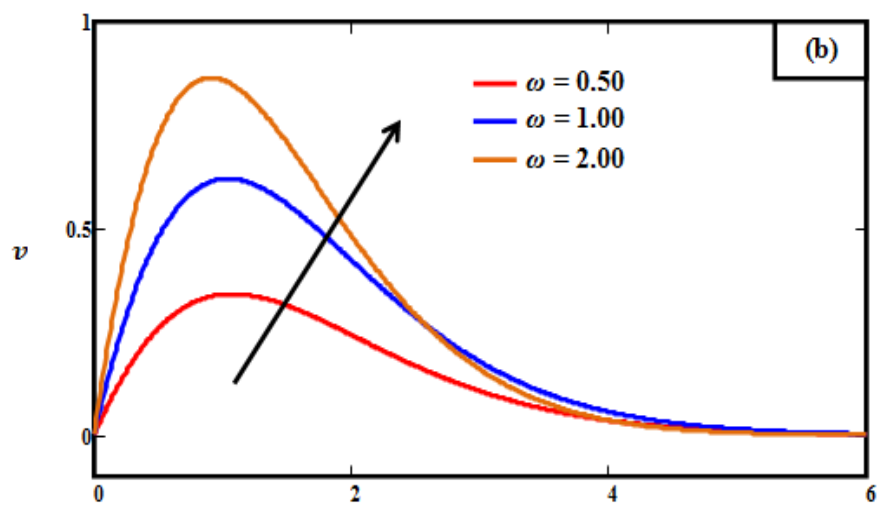

$z$

Fig. 5 Velocity profiles for different values of $\omega$ with $G r=5.0, t=1.0, \alpha=0.2$, $\mathrm{Pr}=0.71$ where (a) primary and (b) secondary velocities.
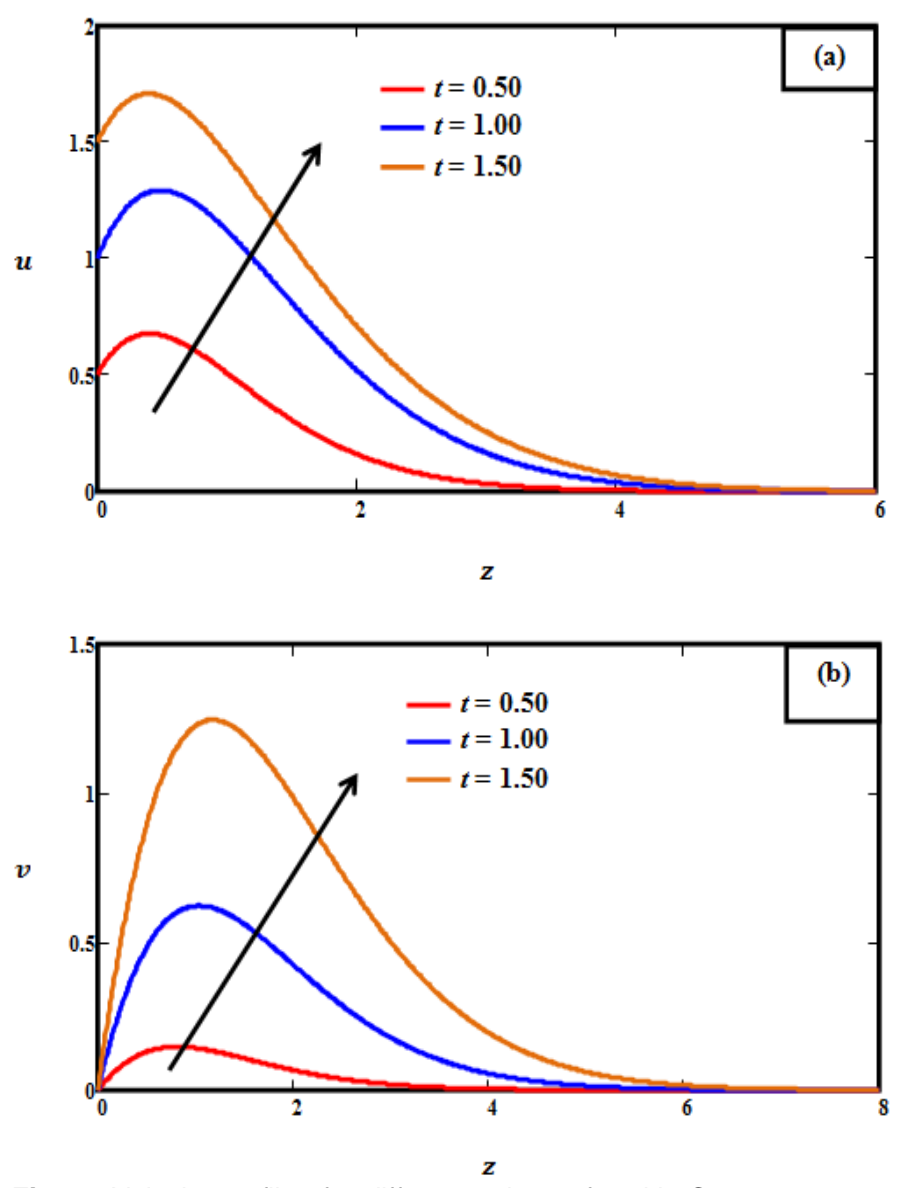

Fig. 6 Velocity profiles for different values of $t$ with $G r=5.0, \omega=1.0$, $\alpha=0.2, \operatorname{Pr}=0.71$ where (a) primary and (b) secondary velocities. 


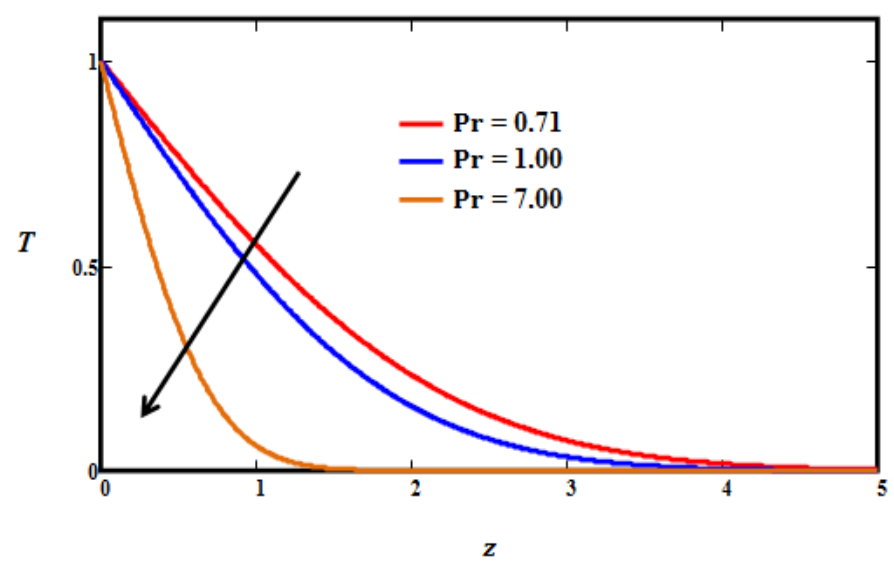

Fig. 7 Temperature profiles for different values of $\operatorname{Pr}$ with $t=1.0$.

\section{CONCLUSION}

In this paper, an exact expression of the rotating and accelerated free convection flows in a second grade fluid was obtained. The dimensionless governing equations were solved by using the Laplace transform technique. Second grade fluid is a sub-class of viscoelastic fluid and it is reasonably hope to obtain exact solutions of the model but this not true for the orthers types of viscoelastic fluid. Thus the exact solutions determined in present study can be applied to verify the results of a more complex viscoelastic fluid model which approximated by using numerical method or pertubation theory. Moreover, the results obtained in this paper showed:

1. Primary velocity increases and secondary velocity initially increases and then decreases decreases while increasing of second grade parameter, $\alpha$.

2. Primary velocity and secondary velocity are incresed by increasing the value of Grashof number, $\mathrm{Gr}$.

3. Primary velocity is enchanced when larger values of rotation parameter, $\omega$ is used. Contrary behaviour is observed for the secondary velocity.

\section{ACKNOWLEDGEMENT}

The authors would like to acknowledge Ministry of Higher Education (MOHE), Research Management Centre of Universiti Teknologi Malaysia (UTM) for the financial support through vote numbers $4 \mathrm{~F} 713,13 \mathrm{H} 74$ and $15 \mathrm{H} 80$ for this research.

\section{REFERENCES}

Hayat, T., Nadeem, S., Siddiqui, A. M., Asghar, S. 2004. An oscillating hydromagnetic non-newtonian flow in a rotating system. Appl Math Lett. 17, 609-614.

Hayat, T., Fetecau, C., Sajid, M. 2008. Analytic solution for MHD transient rotating flow of a second grade fluid in a porous space. Nonlinear Anal. Real World Appl. 9, 1619-1627.

Khan, I., Farhad, A., Sharidan, S., Norzieha, M. 2010. Exact solutions for accelerated flows a rotating second grade fluid in a porous medium. World Appl. Sci. J. 9, 55-68.

Faisal, S., Aziz, Z. A., Ching, D. L. C. 2013. On accelerated MHD flows of second grade fluid in a porous medium and rotating frame. Int. J. Appl. Math. 43, 1-8.

Imran, M. A., Imran, M., Fetecau, C. 2014. MHD oscillating flows of rotating second grade fluids in a porous medium. Communi. Numeric. Analys. 2014, 1-12.

Azhar, A. Z., Vieru, D., Shahraz, A. 2015. Magnetohydrodynamics of rotating fractional second grade fluid in a porous medium. J. Prime Res. Math. 10, 45-58.

Ismail, Z., Khan, I., Mohamad, A. Q., Sharidan, S. 2015. Second grade fluid for rotating MHD of an unsteady free convection flow in a porous medium. Defect Diffus. Forum. 362, 100-107.

Ismail, Z., Khan, I., Awang, R., Nasir, N. M., Salleh, M. Z., Sharidan, S. 2015. Rotation effects on unsteady magnetohydrodynamics second grade fluid flow in a porous medium past an infinite inclined plate. AIP Conf. Proc. $1643,555-562$.

Mohamad, A. Q., Khan, I., Ismail, Z., Sharidan, S. 2016. The unsteady free convection flow of rotating second grade fluid over an oscillating vertical plate. Jurnal Teknologi. 78, 57-63. 